\title{
Diseño y Validación de un Instrumento para Analizar las Representaciones Externas de Estudiantes de Bachillerato sobre Genética
}

\author{
Design and Validation of an Instrument to Analyze High School \\ Student's External Representations about Genetics
}

\author{
Fernando Flores-Camacho \\ Beatriz Eugenia García-Rivera * \\ Araceli Báez-Islas \\ Leticia Gallegos-Cázares
}

Centro de Ciencias Aplicadas y Desarrollo Tecnológico, UNAM

\begin{abstract}
Este trabajo da cuenta de la construcción de un cuestionario como instrumento de investigación para determinar las representaciones de estudiantes de bachillerato sobre el tema de genética, que tiene el propósito de contribuir a los nuevos enfoques en enseñanza de las ciencias centrados en las construcciones representacionales. El instrumento pasó por diferentes criterios de validez (claridad de las preguntas, obtención de respuestas esperadas, ítems pertinentes, inteligibles, con completitud y estructura equivalente), para elaborar la versión final, que fue aplicada a una muestra de 387 alumnos del último curso de Biología del bachillerato de la Universidad Nacional Autónoma de México (UNAM), con la que se obtuvo la confiabilidad del cuestionario (alcanzando un coeficiente alpha de Cronbach de 0.88) y su caracterización con el modelo de crédito parcial de Rasch. Los resultados muestran que se generó un cuestionario confiable para caracterizar diversos tipos de representaciones externas como son respuestas escritas, esquemas y dibujos, lo que evidencia el abanico de las posibles representaciones que tienen los alumnos sobre los diferentes aspectos implicados en el tema de genética, además de que es un instrumento que permite analizar el proceso de transformación de las representaciones iniciales a otras más complejas.
\end{abstract}

Palabras clave: Enseñanza de las ciencias, Evaluación, Representaciones, Bachillerato, Genética.

This paper reports the construction of a questionnaire as a research tool to determine the high school students' representations on genetics' topic, which aims to contribute to the new approaches in teaching science centered on representational constructions. The instrument went through different validity criteria (clarity, obtaining expected answers, pertinent, intelligible, complete and equivalent items) to elaborate the final version, which was applied to a 387 student's sample who were in the last Biology course of High School of the National Autonomous University of Mexico (UNAM), with which the reliability of the questionnaire was obtained (reaching a Cronbach alpha coefficient of 0.88) and its characterization with the Rasch partial credit model. The results show that was generated a reliable questionnaire to characterize diverse types of external representations such as written answers, diagram and drawings, which shows the range of possible representations that the students have about the different aspects involved in genetics issue, besides that it is an instrument that allows to analyze the process of transformation from initial representations to other more complex ones.

Keywords: Science education, Evaluation, Representations, High school, Genetics.

*Contacto: beatrizegr@hotmail.com

issn: 1989-0397

www.rinace.net/riee/

https://revistas.uam.es/riee
Recibido: $\quad 2$ de abril de 2017

$1^{\text {a }}$ Evaluación: 23 de mayo de 2017

Aceptado: $\quad 9$ de junio de 2017 


\section{Introducción ${ }^{1}$}

El presente trabajo describe la construcción de un cuestionario que ha servido como instrumento de investigación del proyecto "Procesos de transformación de las representaciones científicas en los estudiantes del bachillerato bajo un entorno multirepresentacional apoyado con tecnologías digitales", que está centrado en el análisis de las representaciones externas que construyen estudiantes del bachillerato sobre el tema de genética; para ello se construyó, validó y aplicó un cuestionario que sirvió como instrumento de investigación que permite reconocer las diferentes representaciones que construyen los estudiantes de bachillerato. Con el cuestionario se pueden analizar diversas formas descriptivas, interpretativas y explicativas. En este trabajo se reporta el proceso de generación y validación de dicho instrumento.

El desarrollo del instrumento se fundamenta en el enfoque de la construcción de representaciones externas como herramientas cognitivas que permiten a los sujetos organizar y explicitar sus ideas y modelos respecto a una fenomenología particular; lo cual ha abierto un nuevo campo de análisis de los procesos de aprendizaje y exige, por tanto, de la generación de instrumentos que no solo se enfoquen en la explicitación de conceptos o sus interpretaciones, sino además, pueda conocerse el conjunto de elementos que componen sus representaciones explícitas, sean estas expresadas en un lenguaje gráfico, simbólico o en una combinación de ambos y desde las cuales pueden integrar e interpretar una temática como la genética, con lo que es posible dar cuenta de todo un marco de interpretación y comprensión de conceptos y procesos.

Para diferenciar lo que implica un instrumento orientado hacia las representaciones externas pongamos por ejemplo un instrumento de investigación o de evaluación basado en la comprensión de conceptos. En éste, se busca que las preguntas estén dirigidas a que los alumnos enuncien y/o expliciten el concepto de cromosoma, lo que se logra usualmente a partir de preguntas directas, o preguntas donde dicho concepto sea aplicado en una situación o contexto particular (e.g. su relación con el ADN y gen o con el proceso de meiosis y variabilidad genética). Desde un enfoque representacional, la orientación del instrumento se enfoca en que el sujeto explicite no solo un enunciado o relación con una situación, sino también en la forma en que lo relaciona con su experiencia previa, diversas posibilidades para representarlo (descripciones escritas e icónicas), el nivel de organización que el sujeto da al concepto y si estas representaciones externas cambian conforme las tareas lo demandan. Así, en el caso del cromosoma, el sujeto describe el escenario (por ejemplo, dentro de la célula, en cierto momento del ciclo celular) y las formas de representación en las que se apoya (ADN compactado, con una cromátide, con dos cromátides, con genes). Todos estos elementos que en conjunto dan indicios de cómo es el marco representacional desde el cual un sujeto explicita sus ideas.

\footnotetext{
1 Esta investigación forma parte del proyecto "Procesos de transformación de las representaciones científicas en los estudiantes del bachillerato bajo un entorno multirepresentacional apoyado con tecnologías digitales”, financiado por Conacyt con clave: 238712. Los autores agradecen la colaboración de los profesores y estudiantes de la UNAM que participaron en la aplicación del instrumento de evaluación y los valiosos comentarios de la Dra. Elena Calderón-Canales y la Dra. Cynthia Esperanza Lima-González.
} 
Lo anterior hace patente la necesidad de construir instrumentos de investigación acordes con lo que implica la dinámica de un proceso representacional.

La relevancia de la presente propuesta reside en la pertinencia de construir instrumentos de evaluación que sean congruentes con los supuestos y perspectivas de una visión educativa centrada en las representaciones, el cambio representacional y la multirepresentacionalidad. El documento está organizado de la siguiente forma: planteamiento del problema; marco teórico basado en las representaciones y sus implicaciones en el aprendizaje de las ciencias; descripción del proceso de estructuración del instrumento; la metodología seguida para su validación; una muestra de las representaciones de los alumnos analizadas desde el marco propuesto; y consideraciones finales sobre el uso y posibilidades del instrumento.

\subsection{Planteamiento del problema}

En las últimas tres décadas la investigación en el campo del aprendizaje de las ciencias ha pasado por distintas etapas, esto ha llevado a desarrollar nuevos enfoques y formas de enseñanza, que han implicado también una transformación en los procesos e instrumentos de investigación y de evaluación. Un ejemplo relevante de esto es el estudio e identificación de las concepciones alternativas o ideas previas (Duit y Treagust, 1998; Wandersee, Mintzes y Novak, 1994) a partir del cual, se generaron nuevas metodologías e instrumentos para conocer cuáles eran las ideas previas de los estudiantes en los diversos niveles educativos, mostrando que, para el tema aquí tratado, el aprendizaje de la genética es poco significativo y escasamente comprendido por los estudiantes (Ayuso, Banet y Abellán, 1996; Banet y Ayuso, 2000; Bullago, 1995; Cabellero, 2008; Collins y Stewart, 1989; Figini y De Micheli, 2005), quienes muestran dificultades para comprender y representar los conceptos y los procesos implicados, y evidencian también problemáticas asociadas a las estrategias empleadas en el aula para abordar esta temática.

En cuanto al uso de las representaciones, Diez de Tancredi y Caballero (2004) mencionan que en un tema como genética es muy común el uso de representaciones externas por parte de los profesores para presentar la información, esto es recurren a imágenes, por ejemplo, que representen un cromosoma o ADN como apoyo a su explicación verbal. Sin embargo, la mayoría de los instrumentos diseñados para evaluar el conocimiento de los alumnos no considera que el estudiante pueda hacer uso de esas representaciones externas para realizar sus explicaciones. Así, Banet y Ayuso (2000) señalan que es insuficiente contar con instrumentos que atiendan únicamente la explicación o descripción de los conceptos implicados, puesto que un alumno puede dar una respuesta correcta, pero esto no significa necesariamente que el problema o el concepto haya sido comprendido; de hecho, los estudiantes pueden exhibir explicaciones que fueron aprendidas memorísticamente y responder correctamente, sin entender los conceptos que utilizan o los procesos implicados.

Un ejemplo de lo anterior es cuando se utiliza lenguaje gráfico en la clase, como resolver ejercicios de herencia mediante cuadros de Punnett, el significado que los estudiantes le dan generalmente está ligado a su conocimiento cotidiano y no logran identificar las diferencias entre los niveles de representación (e. g. características fenotípicas vs proceso de transcripción) ni la equivalencia entre las explicaciones de las diferentes formas de representación (Galagovsky, Rodríguez, Sanmartí y Morales, 2003). De esta forma, 
pueden resolver sin ningún problema los ejercicios planteados, pero cuando se les cuestiona acerca de qué significan los cruces que realizan, a qué corresponden las letras o cómo se da la expresión de esas características, tienen dificultades para explicar, limitándose únicamente a dar un resultado con las letras que simbolizan los alelos (Ayuso, Banet y Abellán, 1996).

En el proceso de construcción del conocimiento en torno al aprendizaje de las ciencias, a la luz de los nuevos enfoques se ha hecho necesario reconsiderar los procesos de construcción y aprendizaje de los conocimientos científicos en los alumnos y uno de esos procesos de cambio, ha sido el tránsito desde el cambio conceptual hasta la dinámica representacional (Gilbert, 2008; Kozma y Russell, 2005; Pozo 2015). En este enfoque juegan tanto las representaciones internas como las externas y, a partir de ellas, los análisis del aprendizaje de los alumnos se plantean nuevos retos en los niveles teórico y metodológico, y entre ellos los de las formas de poder obtener datos confiables y pertinentes de las representaciones que pueden explicitar los alumnos.

Para atender los cambios en los procesos de construcción y aprendizaje en los alumnos que el enfoque representacional implica, se requieren instrumentos que permitan conocer cómo los estudiantes estan comprendiendo los conceptos y procesos implicados apoyándose en el uso de representaciones externas, pero que además esas representaciones que construyen sirvan para identificar si los conceptos utilizados son comprendidos correctamente o están elaborando representaciones que están alejadas del ámbito escolar. El trabajo aquí descrito pretende apoyar este proceso, con la construcción de un instrumento que permita, a partir de las representaciones externas de los alumnos, analizar sus procesos de comprensión y construcción de conocimiento.

\section{Fundamentación teórica}

\subsection{Representaciones y aprendizaje de las ciencias}

A partir de los trabajos de Duit y Treagust (1998) sobre las ideas previas, surgieron diversas teorías de cambio conceptual con diferentes aproximaciones en los campos epistemológico y cognitivo (Flores, 2004), que ayudaron a mejorar los procesos didácticos y curriculares en la enseñanza de las ciencias (Duschl y Grandy, 2008). A pesar de ello, no se logró una mejoría en la comprensión de los conceptos científicos al nivel que se esperaba. Debido a esto, las posiciones centradas en el cambio a nivel de conceptos se han reconsiderado, centrándose ahora en construcciones más cercanas a lo fenomenológico (elementos cognitivos con los que se interpretan procesos y concepciones), como son las representaciones y su dinámica de cambio en el aprendizaje, donde los conceptos y sus relaciones con otros conceptos, así como diversos elementos fenomenológicos, forman parte de un complejo entramado (Gilbert, 2008; Pozo, 2015).

En este sentido, una representación mental responde a una estructura generada por el sujeto, con la que puede inferir propiedades o cualidades posibles de lo que representa. En esa estructura, y sus posibilidades de hacer predicciones y generar explicaciones, radica su importancia, puesto que se manifiestan elementos útiles para dar cuenta de procesos que podrán o no ser correspondientes con lo observable, pero que satisfacen la coherencia mínima que los sujetos requieren para interpretar y funcionar en su entorno (Flores y Valdez, 2007). 
La generación de recursos cognitivos o de un conjunto representacional pertinente para el aprendizaje de la ciencia, implica el apoyo para que los estudiantes logren desarrollar representaciones mentales de mayor alcance explicativo, esto es, que puedan hacer inferencias con diferentes formas de representación y generen un esquema o modelo representacional con el que puedan comprender el fenómeno desde posibilidades interpretativas diferentes, lo que implicaría, de acuerdo con diSessa (2004), la construcción de nuevas estrategias de lectura y clases coordinadas más amplias.

La construcción y transformación de las representaciones de los estudiantes dentro del ámbito escolar, deben dar cuenta de los elementos conceptuales que ha construido y que les permiten elaborar una interpretación de los fenómenos que se pide comprendan, que muestren indicios de la articulación de sus ideas y de los mecanismos que construyen para darles significado y coherencia. Es por ello que estas representaciones a través de procesos de explicitación, pueden convertirse en objeto de análisis tanto para los alumnos, sus compañeros y para los profesores.

Hay que tomar en cuenta que la explicitación de las representaciones de los alumnos no es una tarea sencilla, ya que implica diferentes niveles de competencias o habilidades para expresarlas. Kozma y Russell (2005) refieren cinco niveles de competencia para la explicitación representacional: Nivel 1, representación como elemento figural; Nivel 2 , nivel básico o primario de habilidad simbólica; Nivel 3, uso sintáctico de representaciones formales; Nivel 4, uso semántico de representaciones formales; Nivel 5, uso reflexivo y retórico de las representaciones. De acuerdo con esto, todos estos niveles de explicitación deberán estar incluidos en un instrumento que pretenda identificar las posibilidades representacionales de los alumnos.

Además de las representaciones que construyen los alumnos, hay que considerar las representaciones externas o externalizadas, que a diferencia de las internas, corresponderían a todo elemento icónico o simbólico con el que se denota a lo que se representa, y que se muestra a los estudiantes como elementos para el aprendizaje y para el pensamiento y que constituyen, según diSessa (2002), el pilar del pensamiento de lo externo al sujeto. Por ejemplo, la representación gráfica de la cadena de doble hélice del material genético es una representación externa lo mismo que la secuencia empleada para distinguir las bases nitrogenadas, o la descripción de su significado. También lo son las representaciones dinámicas como las interacciones y simulaciones o bien, secuencias temporales de imágenes.

Las representaciones externas tienen un importante rol para el aprendizaje (Perkins, 1994) y, como apuntan Prain y Tytler, (2012), contribuyen en la dimensión epistemológica, la cual involucra dos aspectos: los epistémicos, donde la construcción de modelos y variables de las representaciones funcionan como elementos constrictores de los procesos de los sujetos, y la dimensión semántica, donde se dota de significado a símbolos y herramientas simbólicas; es decir, imágenes, esquemas, gráficos o dibujos.

\subsection{Construcción y desarrollo del instrumento de investigación}

Para generar un instrumento que permita hacer explícitas las representaciones de los alumnos, es necesario considerar preguntas o tareas que promuevan los diversos niveles de explicitación (Kozma y Russell, 2005), que estarían en concordancia con lo que se ha denominado Integración de Conocimiento, esto es, 
la habilidad para generar ideas científicamente relevantes y uso de teorías así como de evidencia empírica para conectar ideas en la explicación de fenómenos científicos o justificar proposiciones acerca de un problema científico. El conocimiento integrado se puede caracterizar como un proceso cognitivo dinámico que puede ser capturado o reconocido en las explicaciones. (Lee, Liu y Linn, 2011, p. 116)

Estos mismos autores han mostrado que, cuando se pretende conocer el conocimiento integrado de los alumnos, es decir, que se pueda conocer la comprensión de los conceptos y su uso en situaciones diversas, sus formas de representación externa, así como el nivel de las explicaciones que logran estructurar, los mejores instrumentos son aquellos en los que se pide a los alumnos explicaciones o "ítems de explicación". Sin embargo, estos ítems deben permitir diversos niveles de explicación, esto es, que las respuestas y argumentos que generan para dar cuenta de un ítem puedan tener continuidad en torno a cómo los usan para ampliar o aplicar en otra situación. Atendiendo a estos aspectos de integración, continuidad y extensionalidad, un instrumento de investigacion/evaluación debería considerar en su estructura estas características:

1. Atender a conocimientos que, en principio, han sido analizados a lo largo de las trayectorias escolares de los alumnos, es decir, deben ser temas que atraviesen diversos programas curriculares y ciclos escolares. Esta característica, al considerar los elementos de conocimiento en los que los sujetos han tenido alguna experiencia escolar y extraescolar, concuerda con el enfoque de Integración de Conocimiento, ya que no pretende proponer situaciones nuevas que exijan la construcción de hipótesis que no puedan justificarse de manera amplia y porque se reconoce también que los estudiantes cuentan con un bagaje de representaciones que no podrían poner en juego en temas nuevos o que han sido parcialmente introducidos en el ámbito escolar.

2. Presentar situaciones cotidianas fácilmente interpretables por los estudiantes. Esta característica implica que los sujetos pueden emplear un marco representacional de su entorno y hacer uso explícito de las formas usuales en las que describen esas situaciones.

3. Susceptibles de ser representadas de diversas formas: Verbal, por medio de explicaciones y descripciones; Simbólica, por medio de símbolos matemáticos, ecuaciones u otros símbolos; y Gráfica, por medio de representaciones esquemáticas y de procesos.

4. Posibilitar un proceso de reelaboración de explicaciones a lo largo del instrumento. Esta característica implica el proporcionar a los sujetos diversos momentos de reelaboración de sus representaciones y explicaciones, lo que además de estar de acuerdo con la Integración de Conocimiento, involucra ir construyendo representaciones de mayor complejidad y, con ello, es posible mejorar la apreciación de los niveles que alcanzan los sujetos para explicitar sus construcciones.

Estas características abarcan los elementos necesarios para que, a partir de las respuestas de los sujetos, puedan determinarse patrones de respuesta que den indicios sobre los posibles marcos representacionales con que cuentan los alumnos y las diferencias entre estudiantes que han participado en situaciones escolares diferentes en 
lo referente al uso de diversas formas de representación externa en la escuela como el uso de simuladores, mapas conceptuales, gráficas, etc.

\subsection{Temática de análisis}

De acuerdo con las características y el propósito de este instrumento, es necesario considerar una temática que implique, para su comprensión, diversas formas de representación. Pero, además, que sea una temática que haya sido investigada previamente para tener referentes comparables, que presente cierto nivel de dificultad de comprensión para que el instrumento pueda captar formas de argumentación y descripción y, finalmente que haya sido motivo del currículo de los alumnos.

Dentro de esa caracterización, el tema de genética implica la comprensión del concepto de niveles de organización (bases nitrogenadas, nucleótidos, alelo, gen, cromosoma, ADN, gametos), procesos de meiosis (crossing over) y mitosis, mecanismos de herencia y alteraciones genéticas; cumpliendo con los requisitos planteados, en especial por tratarse de un tema complejo al considerar que estos conceptos tienen un alto grado de abstracción (Diez de Tancredi y Caballero, 2004).

Por ejemplo, se ha identificado que los estudiantes suelen confundir o no reconocer los diferentes niveles de organización, al no ser capaces de representar correctamente ADN, cromosomas, genes y alelos, y usarlos indistintamente como sinónimos (Mills Shaw, Van Horne, Zhang y Boughman, 2008); presentan dificultad para reconocer su estructura y ubicación, por ejemplo, consideran a los genes más grandes que los cromosomas (Caballero, 2008; Iñiguez, 2005; Lewis y Wood-Robinson, 2000; Lewis, Leach y WoodRobinson, 2000), y también tienen problemas en cuanto a la definición del concepto, al referir a gen como una pequeña partícula que lleva el rasgo o característica (Argento 2013; Lewis y Kattmann, 2004). De este modo, si gen y característica son vistos como sinónimos, el alumno no llega a considerar un nivel molecular donde piense en términos de cómo se podría expresar el gen o los procesos implicados, sino que se queda en un nivel macroscópico al pensar que el fenotipo y el genotipo están actuando al mismo nivel, pues no es consciente de su base química, ocasionando así dificultades para relacionar el material hereditario con la síntesis de proteínas y con la expresión de la información genética (Duncan y Reiser, 2007; Duncan, Rogat y Yarder, 2009; MarbachAd, Rotbain y Stavy, 2008; Rotbain, Marbach-Ad y Stavy, 2006). En consecuencia, muchas veces se considera que el ambiente puede influir en la aparición de una característica determinada más que la cuestión hereditaria (Ramagoro y WoodRobinson, 1995), debido a que no se reconocen y distinguen los procesos de meiosis y mitosis, presentando confusión entre ambos o desconociendo su propósito; no logrando relacionar el proceso de meiosis con la formación de gametos ni con la resolución de problemas de genética (Ibáñez y Martínez Aznar, 2005; Lewis, Leach y Wood-Robinson, 2000), es decir, aunque la mayoría de los estudiantes puedan resolver ejercicios de genética mediante cuadros de Punnett, al carecer de un adecuado conocimiento sobre la división meiótica, la segregación de los cromosomas y cómo están relacionados con los símbolos del cuadro, el aprendizaje será mecánico y fragmentado debido a la incapacidad de relacionar los conceptos (Stewart, 1982).

Acorde con esto, para el diseño del instrumento, se contemplaron aquellos aspectos que permitieran identificar los elementos básicos de genética que emplean los alumnos y las 
explicaciones que dan sobre las variaciones y mutaciones o alteraciones, con la finalidad de conocer si los estudiantes tienen la posibilidad de reconocer qué se hereda, quién y qué hereda, y cómo lo hereda. Todo esto, tomando en cuenta que algunos de los principales problemas que tienen los alumnos para comprender la transmisión de la información genética de padres a hijos se relaciona con la forma en que consideran que esta información se codifica, dónde se localiza, cómo se expresa en los individuos, o cuáles son los diferentes niveles en los que se representa, por citar algunos aspectos considerados en la construcción del instrumento.

\subsection{Estructura de los ítems}

Como se ha apuntado, los ítems tienen una estructura orientada a la explicación y, en su construcción, se siguieron las características definidas por Haladyna, Downing y Rodríguez (2002) y Lima (2009) que, si bien presentan recomendaciones para la construcción de ítems de elección múltiple, muchos de los criterios que proponen pueden ser adaptados como preguntas donde haya que justificar la elección hecha o sean en sí mismas preguntas de integración. De entre los principales elementos considerados se tomaron en cuenta: evitar ítems con truco; utilizar un vocabulario simple; poner la idea central en el texto; no usar oraciones en sentido negativo; escribir preguntas en un contexto cotidiano.

7. Observa las siguientes imágenes para que identifiques cómo tienen los lóbulos de la oreja Alicia y Mauricio. Los dos han escuchado que nacer con los lóbulos separados es una característica dominante, pero Mauricio asegura que el bebé puede nacer con los lóbulos unidos como él, porque la madre de Alicia también los tiene unidos (también está su imagen).

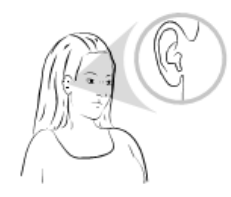

Alicia, lóbulos separados de la mejilla

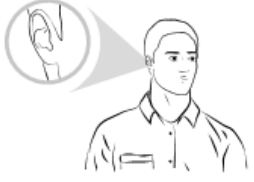

Mauricio, lóbulos unidos a la mejilla

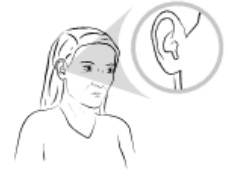

Madre de Alicia, lóbulos unidos a la mejilla

Para ayudarlos a aclarar eso, dibuja cómo imaginas que se presenta la información hereditaria para lóbulos unidos o separados de la mejilla en:

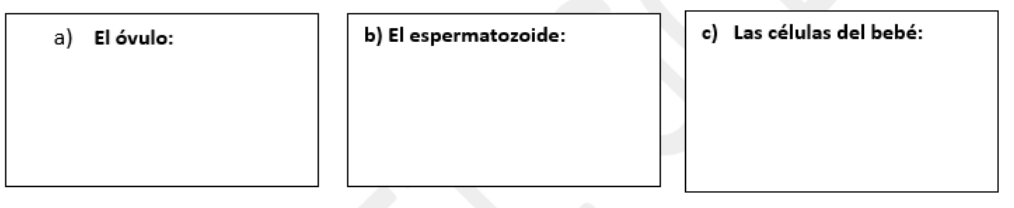

d) Cuando el bebé ya tiene sus lóbulos formados, cen cuáles de sus células está la información para esta característica? ¿Por qué piensas esto?

e) Si solo tómanos en cuenta el genotipo de Alicia y no su fenotipo, ¿qué tan posible es que ella aporte información para que el bebé tenga los lóbulos unidos a la mejilla? ¿Por qué?

f) De acuerdo con la información genética que tiene Mauricio, ¿qué tan probable es que él aporte información para lóbulos unidos a la mejilla? ¿Por qué?

g) De acuerdo con la información que puede aportar cada padre, ¿qué tan probable es que el bebé tenga los lóbulos de la oreja unidos a la mejilla? ¿Por qué?

Figura 1. Los ítems que presenta el cuestionario consideran tanto la elaboración de esquemas o dibujos, como manejo conceptual, resolución de problemas y explicación de

los procesos

Fuente: Elaboración propia. 
Además de las consideraciones descritas, los ítems deben favorecer que los estudiantes expresen diversas formas de representación externa. Por ello los ítems son secuenciados a partir de una problemática que encabeza el cuestionario. A partir de ello, cada ítem implica una demanda donde los sujetos requieren: a) describir en forma escrita explicaciones y descripciones de situaciones; b) elaborar esquemas y dibujos; c) hacer uso de los símbolos gráficos correspondientes al tema; d) relacionar los esquemas realizados con las explicaciones dadas para la construcción de modelos; y e) hacer inferencias a partir de las situaciones planteadas. Por otro lado, la secuencia de los ítems implica mayor nivel de profundidad en las explicaciones por lo que, en su conjunto, el instrumento debe poder dar cuenta del nivel de comprensión del tema científico que se aborda. La figura 1 muestra un ejemplo de los ítems que incluye el cuestionario.

\section{Metodología}

\subsection{Población, contexto y muestra}

El proceso de validación del instrumento siguió tres etapas: Fase de construcción del instrumento, en la cual participaron 10 alumnos de la Facultad de Química y 10 alumnos de bachillerato. Fase de validación de expertos, donde participaron 3 especialistas en Biología y Fase de validación estadística, en la cual participaron 387 estudiantes que cursaban el sexto año del bachillerato en la Escuela Nacional Preparatoria (ENP) y el quinto año en el Colegio de Ciencias y Humanidades (CCH). Los estudiantes de bachillerato que participaron en las distintas fases fueron designados por profesores voluntarios de la ENP y el CCH de la UNAM. Los especialistas en Biología son profesores de la Escuela Nacional Preparatoria y Biólogos que forman parte de este proyecto.

Los estudiantes del bachillerato han llevado cursos de Biología donde se aborda la genética como un tema fundamental del programa del bachillerato de la UNAM. En la ENP se enseña en quinto año (Biología IV) y en sexto año se retoma en el área II (Biología V); mientras que en el CCH se trata en tercer semestre (Biología I) y en quinto semestre (Biología III). En este nivel educativo cuentan además con actividades experimentales que, de una u otra forma, han contribuido a que tengan diversos elementos de representación de un conjunto básico de fenómenos y conceptos científicos.

De los 387 alumnos que conformaron la muestra para la validación estadística del instrumento, el $36 \%$ ha tenido cursos de ciencias en los nuevos laboratorios del bachillerato de la UNAM. Estos laboratorios presentan la posibilidad de que los alumnos exploren además de la experimentación, con diversos recursos digitales como animaciones, uso de simuladores, construcciones de esquemas, videos, consulta en internet y trabajo colaborativo, lo que los hace espacios escolares idóneos para que los alumnos construyan, a través de múltiples representaciones externas, amplios marcos representacionales.

\subsection{Proceso de validación}

El proceso de validación del instrumento con fines de investigación tiene fundamentalmente dos componentes: la validez del instrumento y la confiabilidad del mismo. Ambos criterios son necesarios de cumplir para garantizar que el instrumento mide lo que se espera y que no depende de situaciones específicas de aplicación. 
Después de seleccionar los aspectos sobre los que se preguntaría, se elaboró la primera versión de un cuestionario con la finalidad de tener un conjunto de respuestas lo más detalladas y amplias posibles. Para ello se partió de una situación hipotética, cercana al contexto de los alumnos, de la que se derivaron 11 preguntas (10 de opción múltiple con solicitud de justificación y una abierta) acerca de la formación de un nuevo ser (un bebé humano) y de distintos mecanismos de herencia implicados en dicho evento. No incluyó imágenes de referencia. Este cuestionario se aplicó a 10 alumnos de 2 do semestre de la Facultad de Química de la UNAM. Los resultados obtenidos permitieron identificar la importancia de solicitar la elaboración de un esquema o dibujo en algunos casos (para conocer cómo representaban lo que describían), así como la detección de las preguntas más complejas, mismas que fueron modificadas o sustituidas con lo que se construyó la segunda versión del instrumento.

La segunda versión, fue aplicada a los tres expertos en Biología, esto permitió analizar la inteligibilidad, y plausibilidad de las respuestas, así como suficiencia para abordar el tema y nivel de complejidad de las respuestas esperadas. Entre los resultados obtenidos, se destacó que los dibujos que elaboraban los expertos no aportaban más elementos para comprender sus explicaciones, además de que algunas de sus respuestas no se relacionaban necesariamente con los aspectos que se deseaban analizar. Con ello, se hicieron los ajustes correspondientes y se construyó la tercera versión del instrumento, en la que se incluyeron imágenes de referencia. Además, la información que estos expertos aportaron se tomó como base para la construcción de la rúbrica que sirvió para la asignación de valores como se describirá en la sección de confiabilidad.

Para garantizar que los ítems puedan ser respondidos de manera adecuada y que la información que proporcionan es suficiente para dar una respuesta amplia y correcta desde el punto de vista biológico, la tercera versión del instrumento fue aplicada a 10 alumnos de sexto año de bachillerato del área químico-biológica, que cursaban la materia de Biología V. Esta aplicación se llevó a cabo con la finalidad de afinar las preguntas en cuanto a suficiencia, inteligibilidad, plausibilidad, tiempo de respuesta y completitud de las respuestas. A partir de ese análisis, se corrigieron los últimos detalles de redacción, del diseño de las imágenes de referencia y del orden en que se presentaban las preguntas. Esto llevó a la elaboración de la versión final del cuestionario, conformado por 18 preguntas, algunas de las cuales implicaban respuestas escritas y otras además solicitaban la elaboración de representaciones gráficas. La aplicación a otros 10 estudiantes del nivel mostró que los ítems eran comprendidos de manera suficiente, que proporcionan las ideas de los alumnos de manera clara para poder analizarlos en términos de lo que se espera tanto en los aspectos de múltiple representacionalidad como de comprensión de los conceptos biológicos, por lo que esta versión del cuestionario se definió como el instrumento definitivo para hacer la validación. Con lo anterior se consideran cubiertos los aspectos principales para garantizar la validez del cuestionario de acuerdo a Neumann, Neumann y Nehm (2011).

\subsection{Calificación del instrumento}

Los datos utilizados para las pruebas estadísticas fueron obtenidos a partir de la asignación de valores a las respuestas de los alumnos en los cuestionarios, por lo que para garantizar que esos valores fueran asignados de manera consistente con criterios uniformes, se construyó una rúbrica para calificar las respuestas de los ítems, 
posteriormente se comparó la concordancia de asignación de valor de cada nivel por tres de los investigadores biólogos participantes en el proyecto. La rúbrica utilizada se basa en lo propuesto por Wilson (2005) cuyos criterios se muestra en la tabla 1.

Tabla 1. Criterios para la elaboración de Rúbricas.

\begin{tabular}{|c|c|}
\hline $\begin{array}{l}\text { NIVELES DE INTEGRACIÓN } \\
\text { DE CONOCIMIENTO }\end{array}$ & VALOR Y CARACTERÍSTICAS DE LAS RESPUESTA A LOS ÍTEMS \\
\hline Vínculos complejos & $\begin{array}{l}\text { Valor 5. Explicita tres o más conceptos e ideas relevantes y } \\
\text { elabora dos o más vínculos válidos entre ellas }\end{array}$ \\
\hline Vínculos totales & $\begin{array}{l}\text { Valor 4. Explicita al menos dos conceptos e ideas relevantes y } \\
\text { elabora un vínculo válido entre dos ideas. }\end{array}$ \\
\hline Vínculos parciales & $\begin{array}{l}\text { Valor 3. Explicita ideas o conceptos relevantes pero no elabora } \\
\text { adecuadamente vínculos entre ellos }\end{array}$ \\
\hline No hay Vínculos & Valor 2. Explicita ideas no precisas y relacionadas \\
\hline Irrelevante & Valor 1 . Contiene ideas irrelevantes al contexto científico \\
\hline No hay información & Valor o. No hay respuesta al ítem \\
\hline
\end{tabular}

Con la rúbrica se calificaron los cuestionarios (versión final) y se elaboró la base de datos correspondiente, en la tabla 2 se muestra un ejemplo de la aplicación de la rúbrica con los criterios descritos.

Tabla 2. Ejemplo de aplicación de la rúbrica para asignación de valores.

\section{ÍTEM 13.}

Alicia tiene miedo de que el bebé nazca con síndrome de Down, ayúdala a comprender mejor esta situación, respondiendo si: a) Esto se debe a que hay una alteración genética en la información del bebé. ¿Esto es cierto? ¿Por qué? b) Si consideras que es cierto, haz un dibujo con el que expliques claramente a Alicia dónde se presenta esta alteración y cómo es posible ilustrarla (no olvides describir por escrito lo que representas en tu dibujo)

\begin{tabular}{|c|c|}
\hline Valor O & No contestó \\
\hline Valor 1 & $\begin{array}{l}\text { Irrelevante. La explicación no hace referencia al material genético del sujeto ni de } \\
\text { cómo se ve afectado cuando se presenta el síndrome del ejemplo. }\end{array}$ \\
\hline Valor 2 & $\begin{array}{l}\text { No relacionado. Hace mención a que el síndrome de Down es causado por un } \\
\text { problema genético sin señalar ni hacer referencia del nivel en que se presenta. Los } \\
\text { dibujos que realiza no permiten una clara interpretación de que se relacionan con el } \\
\text { material genético, o hace dibujos sin incluir ninguna explicación por lo que no se } \\
\text { puede inferir si sus dibujos corresponden a cromosomas y en dado caso si están } \\
\text { alterados o están sanos. }\end{array}$ \\
\hline Valor 3 & $\begin{array}{l}\text { Parcialmente relacionado. Menciona que el síndrome de Down es un problema a nivel } \\
\text { del material genético, señalando que es en la cadena de ADN, genes o en los } \\
\text { cromosomas los cuales los dibuja como cortados o deformes, indica que falta o sobra } \\
\text { información, pero no logra precisar con claridad a qué se refiere este síndrome, no } \\
\text { especifica en qué par ocurre la alteración, ni a qué se refiere. También puede hablar de } \\
\text { trisomía, pero sin representar cromosomas o señalar el par } 21 \text {. }\end{array}$ \\
\hline Valor & $\begin{array}{l}\text { Relacionado por completo. Reconoce que el síndrome de Down es resultado de una } \\
\text { alteración a nivel cromosómico, en el que hay un cromosoma de más en el par } 21 \text {, lo } \\
\text { que se le conoce como trisomía } 21 \text {. Los dibujos que presenta hacen referencia a la } \\
\text { presencia de un cromosoma de más en el par } 21 \text {. }\end{array}$ \\
\hline Valor & $\begin{array}{l}\text { Relaciones complejas. Además de las ideas presentes en el nivel anterior, describe } \\
\text { alguna causa que provoca este síndrome como que es durante el proceso de meiosis } \\
\text { donde no hay una correcta separación de los cromosomas homólogos del para } 21 \text {, por } \\
\text { lo que el gameto que se forma se queda con dos, y al unirse con el gameto "sano" } \\
\text { durante la fecundación, habrá } 3 \text { cromosomas en el par } 21 \text {, por lo que el bebé que } \\
\text { resulta de esto presentará síndrome de Down. }\end{array}$ \\
\hline
\end{tabular}

Fuente: Adaptado de Wilson (2005). 


\subsection{Confiabilidad}

Para determinar la confiabilidad del instrumento, la versión final fue aplicada a la muestra de 387 estudiantes de los dos sistemas de bachillerato ya descritos. Se aplicaron dos pruebas estadísticas: la prueba de consistencia interna para lo cual se utiliza el alfa de Cronbach que es una medida interna de la confiabilidad o consistencia interna de un instrumento, y la prueba Modelo de Crédito Parcial de Rasch (Lee, Liu, y Linn, 2011) que determina la discriminación y que se cumpla el propósito y el alineamiento entre los ítems.

\section{Resultados}

La versión final del cuestionario estuvo compuesta por 18 ítems, uno de las cuales fue de opción múltiple y solicitaba justificación, mientras que los 17 restantes eran preguntas abiertas y en 10 de ellas, además de una respuesta escrita, se solicita una representación gráfica. Como ya se mencionó, esta versión fue aplicada a 387 estudiantes de bachillerato y calificada a partir de la rúbrica descrita en la tabla 2.

Los datos obtenidos fueron analizados usando el método de consistencia interna (SPSS) y con el Modelo de Crédito Parcial de Rasch, para el que se empleó el programa Winstep (Bond y Fox, 2015).

El análisis de los resultados muestra que el porcentaje de respuestas que dan cuenta de la Integración del Conocimiento de acuerdo a los niveles 0 a 5 de la rúbrica fueron: $7 \%$ para el nivel 0,3\% para el nivel 1, 19\% para el nivel 2, 22.5\% para el nivel 3, 43\% para el nivel 4 y $5 \%$ para el nivel 5 . De acuerdo a estos resultados, el $65.5 \%$ de los estudiantes de la muestra contestaron en los niveles 3 y 4 .

Con respecto a la confiabilidad, el alpha de Cronbach obtenida para el instrumento diseñado fue de 0.88. Lo que indica una correlación adecuada y buena.

Modelo de crédito parcial de Rasch. Se eligió utilizar este modelo porque, a partir de los niveles de Integración de Conocimiento descritos, permite analizar la medición conjunta y en una misma escala de las habilidades de los sustentantes y las dificultades de los reactivos (González, 2008). La calibración de la muestra utilizada tiene un margen de error de \pm 1 logit. En la tabla 3 se muestran los ítems, organizados por nivel de dificultad a partir del análisis del Modelo de Crédito Parcial de Rasch.

Tabla 3. Resultados del Modelo de Crédito Parcial de Rasch

\begin{tabular}{cccc}
\hline \multicolumn{4}{c}{ Modelo DE CRÉDITO PARCIAL DE RASCH } \\
\hline Ítem & Dificultad & Ítem & Dificultad \\
\hline 6 & 0,426 & 5 & 0,046 \\
8 & 0,408 & 16 & $-0,009$ \\
17 & 0,361 & 14 & $-0,018$ \\
4 & 0,292 & 1 & $-0,030$ \\
7 & 0,278 & 15 & $-0,197$ \\
9 & 0,270 & 10 & $-0,252$ \\
11 & 0,229 & 13 & $-0,566$ \\
12 & 0,146 & 2 & $-0,663$ \\
3 & 0,120 & 18 & $-0,842$ \\
\hline
\end{tabular}

Fuente: Elaboración propia. 
En dicha tabla se aprecia que el rango en la dificultad de los reactivos va de -0.84 a 0.42, donde nueve de los reactivos están por arriba de la dificultad media (0.04) y los ocho restantes por debajo de ella, por lo que se observa que el grado de complejidad entre las preguntas del cuestionario va incrementándose discretamente entre ellas, y no hay alguno que muestre asimetría o se dispare del rango $( \pm 1)$, por lo que se tiene un amplio abanico de las posibles interpretaciones a los problemas planteados. Por ejemplo, el ítem más fácil de responder fue el reactivo 18 (-0.84), mientras que el ítem 6 obtuvo el mayor nivel de dificultad (0.42). Estos datos son consistentes con las estadísticas descriptivas que muestran que el $59 \%$ de la muestra alcanzó el nivel más alto de Integración de Conocimiento en el reactivo 18, con respuestas en el nivel 4 y 5 ; y para el ítem 6 hubo respuestas en todos los niveles de la rúbrica, destacando los niveles o (11\%) y 3 (45\%).

\section{Discusión}

Los resultados obtenidos a partir de las pruebas de confiabilidad y validez, ofrecen el sustento para considerar que se construyó un instrumento confiable para ser aplicado en alumnos del bachillerato pues el valor mínimo aceptable para el coeficiente alfa de Cronbach es 0,70 y el ideal es entre 0,80 y 0,90 (Celina y Campo, 2005; Haertel, 2006). El instrumento desarrollado tuvo un valor de 0.88 , por lo que el constructo cumple con estos requisitos demostrando tener una buena fiabilidad y una validez significativa.

Respecto a la consistencia y confiablidad del instrumento obtenida con el Modelo de Rasch, los ítems resultaron consistentes y existe una medición bastante precisa en cuanto a la dificultad de estos y las habilidades de los estudiantes para resolverlos, pues todos se encuentran dentro del intervalo de 1 a -1, como también han descrito Jiménez y Montero (2013), para un cuestionario de matemáticas.

Queda pendiente aún la cuestión de si el instrumento permite la obtención y posterior evaluación del nivel de representación científica de los alumnos. Desde nuestra perspectiva, el instrumento es efectivo para ese propósito por varias razones:

Las respuestas a los ítems muestran diferentes representaciones externas evidenciadas por la diversidad de conceptos y relaciones que expresan los estudiantes en sus argumentos. En sus respuestas los estudiantes usan argumentos expresados en ítems previos, mostrando con ello que tienen la oportunidad de reflexionar sobre su propio conocimiento y elaborar, sobre su pensamiento inicial, el conjunto base de ideas necesarias para explicar los distintos tipos de interacción. Ejemplo de esto son las explicaciones acerca de la característica que da la forma al lóbulo de la oreja y su probabilidad de expresión, donde se muestra una relación entre los niveles de organización del material genético y los mecanismos de herencia, o bien cuando se pregunta acerca del momento de la fecundación, el estudiante elabora su explicación inicial y adiciona los conceptos de gametos, cromosomas, genes, meiosis y entrecruzamiento. Así, los estudiantes tienen la oportunidad de refinar sus respuestas iniciales, en las que la fecundación es la primera variable considerada, pero se va refinando a lo largo del instrumento con otras de sus respuestas, en las que van relacionando estructuras complejas, y en algunos casos, logran incluir los procesos de mitosis y meiosis. La secuencia de ítems muestra que es adecuada para promover esas oportunidades de refinamiento y elaboración sobre el pensamiento que implica la 
construcción representacional de toda la problemática sobre genética planteada en el instrumento.

Además, se percibe que con el instrumento es posible encontrar diversos niveles que indican a su vez, representaciones de menor o mayor complejidad y claridad en términos conceptuales. Estos niveles de representación se distinguen a partir de las rúbricas y de la correspondencia entre lo que los estudiantes explican, apoyándose en distintos formatos (escrito y simbólico) y lo correspondiente a la ciencia escolar (también con formatos escritos y simbólicos). En la tabla 4 se muestra el caso de tres sujetos que presentan distintos niveles de pertinencia en sus explicaciones y las figuras dan cuenta de sus representaciones sobre el síndrome de Down.

Tabla 4. Descripción de la comparación entre las representaciones en el ítem 13.

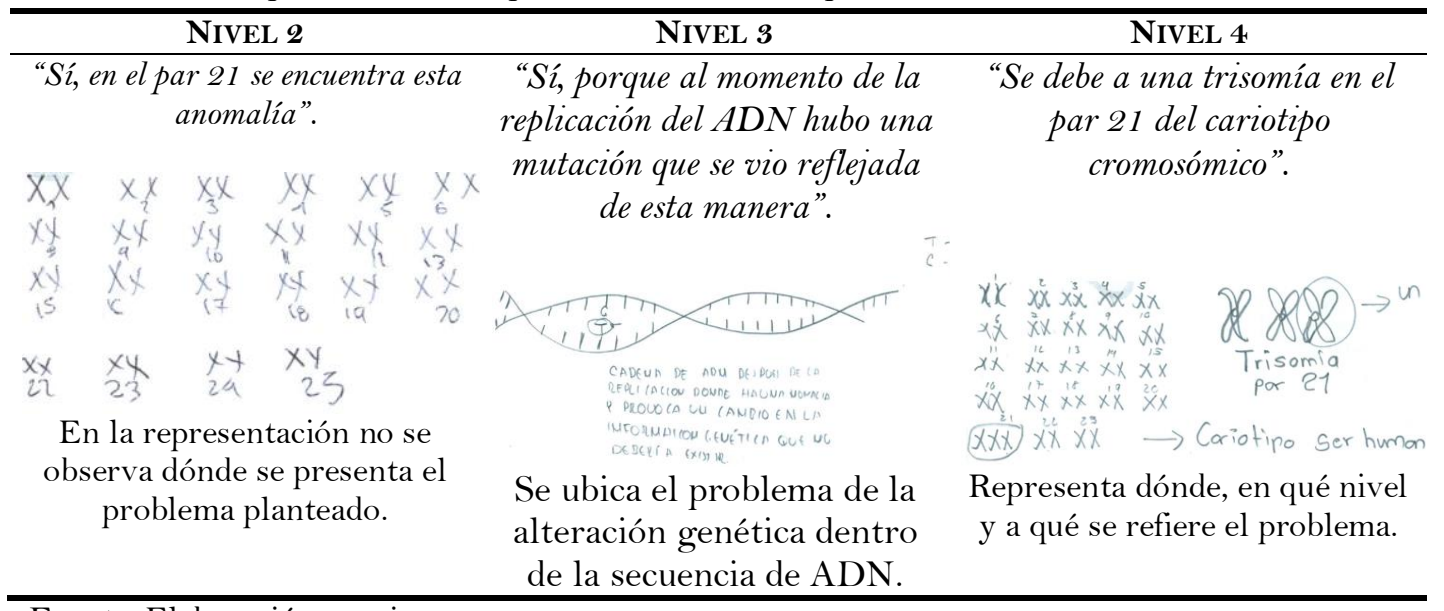

Fuente: Elaboración propia.

Como puede observarse, el instrumento permite conocer las representaciones de los alumnos y analizar cómo algunos conceptos o procesos, aunque son definidos correctamente, cuando se solicita al estudiante que los represente gráficamente, sus representaciones externas no siempre son correctas o no corresponden con lo solicitado, lo que tiene que ver con el nivel de abstracción de determinados conceptos o procesos. Por ejemplo, como se mencionó en los resultados, el ítem 6 fue el más difícil de responder; y en él se solicita representar la información genética que existe en una célula de la piel y en un espermatozoide. Las respuestas de los alumnos muestran que, a pesar de que en el ítem anterior contestaron correctamente que todas las células del cuerpo tienen la misma información genética y que los gametos tienen la mitad de esa información, en sus representaciones son incapaces de describir cómo esa mitad de información se encuentra en los gametos, o la representan únicamente como cromosomas sexuales (XY), e incluso hay quienes no reconocen que exista material genético en las células de la piel, pues solo dibujan organelos.

\section{Conclusiones}

El presente trabajo es una alternativa para la construcción de instrumentos adecuados para determinar y evaluar las representaciones de los sujetos en temas de genética que proporcionen, además de los elementos de comprensión, las posibilidades interpretativas que tienen los alumnos para dar cuenta de temas y conceptos científicos y, con ello, tener 
mejores parámetros en el campo de investigación para seguir el proceso de construcción y, en el contexto escolar para evaluar su conocimiento. El instrumento descrito puede ser útil para relacionar cómo los alumnos representan (e interpretan) los esquemas usuales de los libros de texto con la comprensión de los conceptos y, sobre todo, cómo construyen, ante una situación biológica posible (mecanismos de herencia), un escenario compuesto de un marco representacional a partir de elementos cotidianos y de la ciencia escolar, donde describir sus posibles explicaciones e inferencias.

En síntesis, este cuestionario nos permite conocer las posibilidades que los alumnos tienen para responder preguntas con descripciones escritas y gráficas, a la par que nos sirve para identificar, a través de sus representaciones explícitas, su comprensión de procesos y relaciones para explicar los mecanismos de herencia, con lo que se va más allá de una descripción conceptual que, en la mayoría de las veces, puede ser memorística. Por ejemplo, un problema recurrente que se identificó es que, aunque puedan dar una definición correcta del cromosoma, cuando lo representan gráficamente lo ubican dentro de la cadena de ADN, lo que derivara en dificultades para comprender las interacciones entre los distintos componentes del material genético para la transmisión de las características de los padres a la descendencia.

Es claro que el instrumento desarrollado no es más que una propuesta que puede orientar el desarrollo de instrumentos equivalentes en otros temas de Biología y otras ciencias, pero consideramos que puede ser relevante al reciente enfoque representacional en la investigación y la enseñanza de las ciencias pues los resultados aquí descritos sugieren que, para una plena comprensión de un tema como el de Genética, no es suficiente indagar solo la parte conceptual, se debe además promover el uso y explicitación de las representaciones de los alumnos para identificar cómo interpretan y comprenden sus procesos y conceptos.

Desde luego que esta situación no se puede generar si los alumnos no saben cómo explicitar esas representaciones, por lo que este trabajo abre un abanico de posibilidades sobre cómo se debe promover la explicitación de las representaciones en los estudiantes y cómo, al utilizar este tipo de instrumentos, es posible realizar investigaciones donde se analicen los distintos niveles de complejidad representacional que contribuyan a una mejor comprensión de los procesos cognitivos y de comprensión de los alumnos.

\section{Referencias}

Argento, D. (2013). Estudio exploratorio sobre preconcepciones en el área de Genética en alumnos de secundaria italianos y españoles. Tesis de máster. Universidad internacional de La Rioja, Madrid.

Ayuso, E., Banet, E. y Abellán, T. (1996). Introducción a la genética en la enseñanza secundaria y el bachillerato: II. ¿Resolución de problemas o realización de ejercicios?. Enseñanza de las Ciencias, 14(2), 127-142.

Banet, E. y Ayuso, E. (2000). Teaching Genetics at Secondary School: A Strategy for Teaching about the Location of Inheritance Information. Science Education, 84, 313-351.

Bond, T. y Fox C. (2015). Applying the Rasch Model: Fundamental Measurement in the Human Sciences. Nueva York, NY: Routledge. 
Bugallo Rodríguez, A. (1995). La didáctica de la genética: revisión bibliográfica. Enseñanza de las Ciencias, 13, 379-385.

Caballero, M. (2008). Algunas ideas del alumnado de secundaria sobre conceptos básicos de genética. Enseñanza de las Ciencias, 26(2), 227-243.

Celina, H. y Campo, A. (2005). Aproximación al uso del coeficiente alfa de Cronbach. Revista Colombiana de Psiquiatría, 34(004), 572-580.

Collins, A. y Stewart, J. (1989). The Knowledge Structure of Mendelian Genetics. The American Biology Teacher, 51(3), 143-149.

Diez de Tancredi, D. y Caballero, C. (2004). Representaciones externas de los conceptos biológicos de gen y cromosoma. Su aprendizaje significativo. Revista de Investigación, 56, 91-121.

diSessa, A. A. (2002). Why "conceptual ecology" es a good idea. En M. Limón y L. Mason (Eds.), Reconsidering Conceptual Change: Issues in Theory and Practice (pp. 29-60). Dordrecht: Kluwer Academic Publishers.

diSessa, A. (2004). Coordination and contextuality in conceptual change. En E. F. Redish y M. Vincentini (Eds.), Research on Physics Education: Proceedings of the International School of Physics "Enrico Fermi" (pp. 137-15). Amsterdam: 6IOS Press.

Duit, R. y Treagust, D. F. (1998). Learning in science- from behaviourism towards social constructivism and beyond. En B. Fraser y K. Tobin (Eds.), International handbook of science education, Part 1 (pp. 3-25). Dordrecht: Kluwer.

Duncan, R.G., Rogat, A.D. y Yarder, A. (2009). A learning progression for deepening students' understandings of modern genetics across the 5th-10th grades. Journal of Research in Science Teaching, 46(6), 655-674. https://doi.org/10.1002/tea.20312

Duncan, R. G. y Reiser, B. J. (2007). Reasoning across ontologically distinct levels: students' understandings of molecular genetics. Journal of Research in Science Teaching, 44(7), 938959. https://doi.org/10.1002/tea.20186

Duschl, R. y Grandy, R. (2008). Reconsidering the character and role of inquiry in school science: Framing the debates. En R. Duschl y R. Grandy (Eds.), Teaching Scientific Inquiry: Recommendations for Research and Implementation (pp. 1-37). Rotterdam: Sense Publishers.

Figini, E. y Micheli, A. (2005). La Enseñanza de la Genética en el nivel medio y la educación polimodal: contenidos conceptuales en las actividades de los libros de texto. Enseñanza de las Ciencias, extra (VII Congreso), 1-5.

Flores, C. F. (2004). El cambio conceptual: interpretaciones, transformaciones y perspectivas. Educación Química, 15, 256-269.

Flores, C. F. y Valdez, R. (2007). Enfoques epistemológicos y cambios representacionales y conceptuales. En J. Pozo y F. Flores (Eds.), Cambio conceptual y representacional en el aprendizaje y la enseñanza de la ciencia (pp. 21-35). Madrid: Antonio Machado Libros.

Flores, C. F. y Gallegos, C. L. (1999). Construcción de conceptos físicos en estudiantes. La influencia del contexto. Perfiles Educativos, XXI(85), 90-103.

Flores-Camacho, F., Gallegos-Cázares, L., Garritz, A, y García-Franco, A. (2007). Incommensurability and multiple models: Representations of the structure of matter in undergraduate chemistry students. Science $\&$ Education, 16, 775-800. https://doi.org/10.1007/s 11 191-006-9049-3 
Galagovsky, L., Rodríguez, M., Sanmartí, N. y Morales, L. (2003). Representaciones mentales, lenguajes y códigos en la enseñanza de ciencias naturales. Un ejemplo para el aprendizaje del concepto de reacción química a partir del concepto de mezcla. Enseñanza de las Ciencias, 21(1), 107-121.

Gilbert, J. (2008) Visualization: an emergent field of practice and enquiry in science education. En J. K., Gilbert, M. Reiner y M. Nakhleh (Eds.), Visualization: Theory and practice in science education (pp.3-24). Amsterdam: Springer.

González, M. (2008). El Análisis de Reactivos con el Modelo Rasch, Manual Técnico A. Serie: Medición y Metodología. Ciudad de México: Universidad de Sonora.

Haladyna, T., Downing, S. M. y Rodríguez, M. C. (2002). A review of multiple-choice itemwriting guidelines for classroom assessment. Applied Measurement in Education, 15(3), 309333.

Haertel, E. (2006) Reliability. En R. Brennan (Ed.), Educational measurement (pp. 65-110). Westport, CT: American Council on Education.

Ibáñez, T., y Martínez Aznar, M. (2005). Solving problems in Genetic II: Conceptual restructuring. International Journal of Science Education, 27(12), 1495-1519. https://doi.org/10.1080/09500690500186584

Iñiguez, J. (2005). La enseñanza de la genética, una propuesta didáctica para la educación secundaria obligatoria desde una perspectiva constructivista. Tesis doctoral. Universidad de Barcelona, Barcelona.

Jiménez, K. y Montero, E. (2013). Aplicación del modelo de Rasch en el análisis psicométrico de una prueba de diagnóstico en matemática. Revista digital Matemática, Educación e Internet, $13(1), 1-24$.

Kozma, R. y Russell, J. (2005) Students becoming chemists: developing representational competence. En J. Gilbert (Ed.), Visualization in Science Education (pp. 121-146). Amsterdam: Springer.

Lee, H-S., Liu, O, L. y Linn, M. (2011) Validating measurement of knowledge integration in science using multiple-choice and explanation items. Applied Measurement in Education, 24(2), 115-136. https://doi.org/10.1080/08957347.2011.554604.

Lewis, J., Leach J. y Wood-Robinson, C. (2000). All in the genes? Young people's understanding of the nature of genes. Journal of Biological Education, 34(2), 74-79. https://doi.org/10.1080/002 19266.2000.9655689

Lewis, J., y Kattmann, U. (2004). Traits, genes, particles and information: re-visiting students' understandings of genetics. International Journal of Science Education, 26(2), 195-206. https://doi.org/10.1080/0950069032000072782

Lewis, J., y Wood-Robinson, C. (2000). Genes, chromosomes, cell division and inheritance: do students see any relationship? International Journal of Science Education, 22(2), 177-195. https://doi.org/10.1080/095006900289949

Lima C. E (2009). Changes in state of matter: A study of validity of Texas math and science diagnostic system. Master Thesis, Texas University, Austin.

Marbach-Ad. G., Rotbain, Y. y Stavy, R. (2008). Using computer animation and illustration to improve High School students' achievement in Molecular Genetics. Journal of Research in Science Teaching, 45(3), 273-292. https://doi.org/10.1002/tea.20222 
Mills Shaw, K. R., Van Horne, K., Zhang, H. y Boughman, J. (2008). Essay Contest Reveals Misconceptions of High School Students in Genetics Content. Genetics, 178(3), 1157-1168. https://doi.org/10.1534/genetics.107.084194

Neumann, I., Neumann, K. y Nehm, R. (2011). Evaluating instrument quality in science education: Rasch-based analyses of a nature of science test. International Journal of Science Education, 33(10), 1375-1405. https://doi.org/10.1080/09500693.2010.511297

Perkins, D. (1994). A new look in representations for mathematics and science learning. Instructional Science, 22, 1-37.

Pozo, J. I. (2015). Aprendizaje de la ciencia mediante múltiples sistemas de representación. En F. Flores (Coord.), Las tecnologías digitales en la enseñanza experimental de las ciencias, fundamentos cognitivos y procesos (pp. 13-31). Ciudad de México: UNAM-Porrúa.

Prain, V. y Tytler, R. (2012). Learning through constructin representation in science: A framework or represenational construction affordances. International Journal of Science Education, 34(17), 2751-2773. https://doi.org/10.1080/09500693.2011.626462

Ramagoro, G. y Wood-Robinson, C. (1995). Botswana children's understanding of biological inheritance. Journal of Biological Education, 29(1), 60-71.

Rotbain, Y., Marbach-Ad, G. y Stavy, R. (2006). Effect of bead and illustrations models on High School students' achievement in Molecular Genetics. Journal of Research in Science Teaching, 43(5), 500-529. https://doi.org/10.1002/tea.20144

Stewart, J. (1982). Difficulties experienced by High School students when learning basic Mendelian Genetics. The American Biology Teacher, 44(2), 80-89.

Wandersee, J., Mintzes, J., y Novak, J., 1994, Research on alternative conceptions in science. En D. Gabel (Ed.), Handbook of research on science teaching and learning (pp. 177-210). Nueva York, NY: Mcmillan Publishing Company.

Wilson, M. (2005). Constructing measures: An item response modeling approach. Mahwah, NJ: Lawrence Erlbaum Associates.

\section{Breve CV de los autores}

\section{Fernando Flores-Camacho}

Su campo de trabajo es la enseñanza de la ciencia. Es autor de diversos artículos en sus líneas de investigación como cambio conceptual y representacional, concepciones de ciencia y estudios de la construcción de conocimientos científicos en culturas diversas. También ha sido autor de libros y capítulos en libros sobre educación en ciencia y de texto de Física. Ha sido profesor de la carrera de Física y del Posgrado de Pedagogía de la UNAM. Email: fernando.flores@ccadet.unam.mx

\section{Beatriz Eugenia García-Rivera}

Bióloga, por la Facultad de Ciencias de la UNAM y Maestra en Pedagogía por la Facultad de Filosofía y Letras de dicha institución, donde actualmenteestudia el Doctorado en Pedagogía. Ha participado en distintos proyectos dentro del Grupo de Cognición y Didáctica de la Ciencia del CCADET-UNAM, como "Procesos de transformación de las representaciones científicas en los estudiantes del bachillerato bajo 
un entorno multirepresentacional apoyado con tecnologías digitales" y "Laboratorios de Ciencias para el Bachillerato, UNAM”. Coautora de artículos de investigación, como "Construcción de una secuencia didáctica sobre catabolismo para el laboratorio de ciencias del bachillerato UNAM". Ha impartido diversos cursos a profesores de primaria, secundaria y Bachillerato. Email: beatrizegr@hotmail.com

\section{Araceli Báez-Islas}

Bióloga, por la Facultad de Estudios Superiores Zaragoza y Maestra en Docencia para la Educación Media Superior-Biología por la Facultad de Ciencias de la UNAM. Ha participado en distintos proyectos en materia ambiental dentro del Instituto de Investigaciones Forestales Agrícolas y Pecuarias dentro del Grupo de Geomática, como "Riesgo del hábitat invernal de la mariposa monarca por Scolytus mundus y cambio climático", así como en proyectos de educación como "Procesos de transformación de las representaciones científicas en los estudiantes del bachillerato bajo un entorno multirepresentacional apoyado con tecnologías digitales". Coautora de artículos de investigación, como "Riesgo del hábitat de la mariposa monarca (Danaus plexippus) ante escenarios de cambio climático". Ha impartido cursos a profesores de Bachillerato. Email: baezi.araceli@gmail.com

\section{Leticia Gallegos-Cázares}

Física de formación, Maestra en Enseñanza Superiror y Doctora en Pegagogía. Trabaja desde hace más de veinte años en el campo de la enseñanza de la ciencia. Autora de más de 30 artículos de investigación sobre aspectos de formación conceptual, cambio conceptual y concepciones de ciencia de los profesores en revistas internacionales y nacionales con arbitraje, así como autora y coautora de diversos libros y capítulos en libros sobre educación en ciencia, y libros de texto de Física para el nivel de secundaria. Ha participado en diversas investigaciones apoyadas por la UNAM, el CONACYT, el ILCE y la SEP, algunos de esos proyectos están relacionados con el conocimiento de los mecanismos de construcción de las representaciones conceptuales en la ciencia en alumnos de bachillerato y universidad. Email: leticia.gallegos@ccadet.unam.mx 